\title{
A heuristic for placement of limited range wavelength converters in all-optical networks
}

\author{
K.R. Venugopal *, M. ShivaKumar, P. Sreenivasa Kumar \\ Department of Computer Science and Engineering, Indian Institute of Technology Madras, Chennai 600 036, India
}

Received 21 July 1998; received in revised form 11 May 2000; accepted 29 May 2000

\begin{abstract}
Wavelength routed optical networks have emerged as a technology that can effectively utilize the enormous bandwidth of the optical fiber. Wavelength converters play an important role in enhancing the fiber utilization and reducing the overall call blocking probability of the network. As the distortion of the optical signal increases with the increase in the range of wavelength conversion in optical wavelength converters, limited range wavelength conversion assumes importance. Placement of wavelength converters is a NP complete problem [K.C. Lee, V.O.K. Li, IEEE J. Lightwave Technol. 11 (1993) 962-970] in an arbitrary mesh network. In this paper, we investigate heuristics for placing limited range wavelength converters in arbitrary mesh wavelength routed optical networks. The objective is to achieve near optimal placement of limited range wavelength converters resulting in reduced blocking probabilities and low distortion of the optical signal. The proposed heuristic is to place limited range wavelength converters at the most congested nodes, nodes which lie on the long lightpaths and nodes where conversion of optical signals is significantly high. We observe that limited range converters at few nodes can provide almost the entire improvement in the blocking probability as the full range wavelength converters placed at all the nodes. Congestion control in the network is brought about by dynamically adjusting the weights of the channels in the link thereby balancing the load and reducing the average delay of the traffic in the entire network. Simulations have been carried out on a 12-node ring network, 14-node NSFNET, 19-node European Optical Network (EON), 28-node US long haul network, hypothetical 30-node INET network and the results agree with the analysis. (c) 2001 Elsevier Science B.V. All rights reserved.
\end{abstract}

Keywords: Wavelength converters; WDM networks; Lightpaths; Congestion control; Adjacent wavelength conversion

\section{Introduction}

WDM wavelength convertible optical networks have potential to meet the exponential growth in

\footnotetext{
${ }^{*}$ Corresponding author. Department of Computer Science and Engineering, University Visvesvaraya College of Engineering, Bangalore University, Bangalore, India. Tel.: +91-5257818.

E-mail addresses: rajuk@aries.iitm.ernet.in, rajuk@bgl. vsnl.net.in (K.R. Venugopal), psk@iitm.ernet.in (P. Sreenivasa Kumar).
}

the user traffic catering to the needs of divergent requirements such as high bandwidth online medical applications, real-time rocket and satellite communications, secure transaction processing in federated databases, defense applications, multimedia traffic, supercomputer interconnects along with smaller bandwidth requirements of voice, data and many other applications to millions of users [2]. The enormous mismatch between the low loss usable optical fiber bandwidth $(50 \mathrm{THz})$ and the peak electronic speed $(10 \mathrm{~Gb} / \mathrm{s})$ has led to the 
phenomenal growth of the WDM technology. Wavelength routed networks have evoked keen interest as they are able to provide improved network capacity, reliability, simple network management and independence from modulation format and bit rate. The optical transmission bandwidth is split into a number of non-interfering and non-overlapping wavelength communication channels each operating asynchronously on a different wavelength and at different bit rates throwing challenges to design and develop suitable network architectures, protocols and algorithms [3]. An all-optical wavelength routed network allows connections to be set up between arbitrary source-destination pairs without the signal undergoing opto-electronic conversion at the intermediate nodes. Wavelength routing provides the network with the ability to localize information flow, thereby allowing the same wavelength to be reused in spatially disjoint segments of the network. This capability is of paramount importance in the design of wide area all-optical networks $[4,5]$.

The WDM photonic network consists of wavelength routing nodes interconnected by a pair of unidirectional optical fibers. The wavelength routing nodes are capable of switching the optical signals dynamically based on their wavelength on the arrival of the signal at the input port [6]. These routing nodes are composed of a set of wavelength multiplexers, photonic switches and wavelength demultiplexers. The wavelength routing nodes can be classified into (i) nodes with wavelength conversion capabilities which can convert an incoming optical signal on a wavelength to another outgoing wavelength and (ii) nodes without wavelength conversion capabilities in which the incoming and outgoing optical signals are on the same wavelength $[7,8]$.

A lightpath connection between two nodes along a route is an optical path with or without wavelength conversion. If a connection is set up using only a single wavelength on all the links along the path, then it satisfies wavelength continuity constraint. Wavelength routed optical networks with converters have been able to address the problem of capacity utilization, failure recovery and scalability. It is possible to assign wave- lengths on a link-by-link basis thereby relaxing the wavelength continuity constraint. It facilitates nodes to overcome link and channel failures by allowing local rather than global reconfiguration in the network. It is of interest to note that wavelength converters are very expensive and at present all-optical converters are only capable of limited range wavelength conversion. In light of these optical constraints, researchers have tried to focus on the algorithms for placement of limited range wavelength converters.

This paper explores the impact of limited range wavelength converters vis-a-vis the full range wavelength converters and proposes a heuristic for determining the placement of limited range wavelength converters at a subset of the nodes of the network in order to reduce signal distortion. An analytical model is derived for the placement of limited range wavelength converters in a ring. We have proposed a heuristic algorithm to place limited range wavelength converters in arbitrary mesh networks at a limited number of nodes and have shown that the performance is almost the same as that of a network with full range wavelength conversions at all the nodes. The routing technique reduces the number of cascaded wavelength conversions and the wavelength assignment algorithm limits the range of the wavelength conversion enhancing the optical signal quality. The average delay, congestion and blocking probability is improved by using the dynamically varying state of the network.

The paper is organized into the following sections. Section 2 presents the wavelength converter technology, its components and a review of related work. The network model and the auxiliary graph is described in Section 3. Section 4 addresses the problem of placement of limited range wavelength converters, congestion control and blocking probability in a ring and arbitrary mesh networks. An analytical model for optimal placement of limited range wavelength converters is developed for a ring network in Section 5. Algorithms for the problem are provided in Section 6. Simulation and example networks are given in Section 7. Numerical results are presented in Section 8. Section 9 contains the conclusions. 


\section{Related work}

A wavelength converter can translate the optical signal on one wavelength at the input port to another wavelength at the output port. Wavelength converters increase the complexity and cost of the switch in addition to the protocols and algorithms required to manage them [9-11]. Wavelength converter can be dedicated to each channel, or can be shared by a group of channels to reduce the cost [1]. A dedicated converter bank can be provided to each link or to each node. There are two types of wavelength converters: Optoelectronic converters and all-optical converters. In opto-electronic wavelength conversion, the signal is converted into the electronic form and the resulting signal is modulated on a different wavelength, but the advantage of the alloptical transmission and switching is sacrificed [12]. In all-optical wavelength converters, wavelength conversion from input to output wavelength remains entirely in the optical domain. They can be classified into three groups, (i) gain saturation amplifier based on Injection current [13]; (ii) Coherent converters: Four Wave Mixing (FWM) [14] and Semiconductor Optical Amplifiers (SOAs) [15] (iii) optical control gate converters [16].

FWM converters are transparent to signal format, operate at high bit rates and convert several WDM channels simultaneously $[14,15,17]$, but their conversion efficiency is low $(-20 \mathrm{~dB})$ and hence the $\mathrm{S} / \mathrm{N}$ ratio of the converted optical signals needs to be improved especially if converters are to be cascaded. The most promising is the optical control gate converter. It is extremely simple, polarization insensitive and power efficient $[16,18]$. Wavelength converters increase wavelength reuse which is dependent on four factors, namely, the network size and topology, the traffic distribution in the network, the number of wavelengths, and the Routing and Wavelength Assignment algorithm [19]. A particularly interesting analysis about wavelength reuse factor is available in $[19,20]$. Wavelength convertible networks support higher loads. Extensive study of load in ring networks are available in [21-25].
The impact of wavelength converters on the performance of wavelength routed networks has been explored by simulation and various analytical models. In [1], a shortest path routing algorithm is used to reduce the number of converters leading to the concept of share-pernode or share-per-link wavelength convertible switch, but the complexity of the algorithm is $\mathrm{O}\left(n^{4} w^{2}\right)$, where $n$ is the number of wavelength routers and $w$ is the number of wavelengths per fiber link. A faster algorithm of $\mathrm{O}\left(n^{2} w^{2}\right)$ is developed in [26]. Ramaswami and Sivarajan [19] have derived a lower bound for blocking probabilities with and without wavelength converters. In [27], a generalized reduced load approximation scheme has been used to compute the blocking probabilities for the optical network employing fixed routing for arbitrary topology with path lengths of at most three hops. It is accurate and computationally intensive and therefore tractable only for networks with a small number of nodes.

Chlamtac et al. [28] have presented an efficient algorithm in $\mathrm{O}[(k+n) k n]$ time complexity, where $k$ is the number of wavelengths per fiber and $n$ is the number of nodes to optimally route lightpaths taking link and wavelength conversion costs into consideration. Barry and Humblet [29] have derived expressions for blocking probabilities in paths with and without wavelength converters and examined the effects of path length, number of wavelengths and switch size on the network performance. Similarly, in [20], approximate analytical expressions for overall blocking probabilities have been derived for various network topologies. Barry and Humblet [30] have derived a lower bound on the number of switching states in a network with wavelength converters. In [31], it is shown that wavelength converters causes insignificant reduction in blocking probability at light loads, whereas at medium loads, the gain is significant. Alternate routing with wavelength converters reduces blocking probability, but introduces network instability [32].

Subramaniam et al. [33] have presented a probabilistic model to estimate the performance of optical networks with sparse wavelength conver- 
sion. In [34], an attempt is made to study the effect of wavelength conversion under dynamic nonPoisson traffic input. The model predicts that traffic peakedness plays a critical role in determining the blocking performance while the wavelength conversion is insensitive to traffic peakedness over a large range. Gerstel et al. $[22,35]$ were first to examine limited wavelength conversion for ring networks under a non-probabilistic model. In [25], a non-probabilistic analysis for tree networks and networks of arbitrary topology has been presented.

Multifiber solution as an alternative to wavelength conversion has been explored in [36,37]. The analysis is an extension of the work in [29]. In this approach, the number of fibers to be minimized are more important than the number of wavelengths. In [38], limited wavelength conversion based on FWM has been investigated. It assumes link load and wavelength independence. The conversion efficiency drops with increasing range. The wavelength conversion with as little as one-fourth the full range gives a good performance while half of the full range wavelength conversion delivers almost all of the performance improvement. In [39], placement of wavelength converters in a path under uniform and non-uniform loads has been investigated. Sharma and Varvarigos [40] have studied limited range wavelength conversion for mesh networks over a wide range of network loads.

\section{Network model}

\subsection{Definitions}

- The Physical topology (Fig. 1) consists of wavelength routing nodes interconnected by a pair of unidirectional fiber links.

- A lightpath is established by the allocation of a wavelength on each link of the path between the source and the destination.

- The virtual topology is the set of lightpaths established between the nodes of the network.

- Same wavelength can be used in the disjoint paths of the network and is termed as Wavelength Reuse.

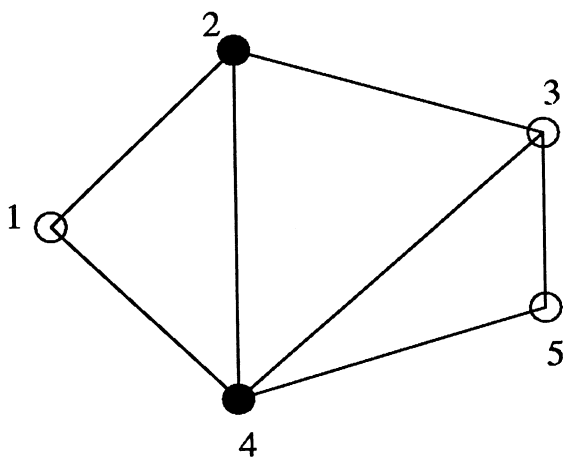

Fig. 1. Physical topology.

- Blocking probability is the ratio of the number of lightpath requests rejected to the number of lightpath connections requested.

- Load is the rate of connection requests per unit time. One Erlang load is the number of calls per unit call holding time.

- Congestion is the utilization of the heaviest loaded link in the network.

- Realizing a virtual topology for a set of lightpaths known apriori is called static lightpath establishment (SLE).

- Determining a route for the lightpath requests that arrive in real time without affecting the existing lightpaths is called dynamic lightpath establishment (DLE).

- The wavelength conversion gain is the ratio of the difference in the blocking probabilities with and without wavelength converters and the blocking probability without wavelength converters.

\subsection{Physical network as a graph}

Let $G_{\mathrm{p}}=(V, E)$ be a graph representing the physical network topology and $E \subseteq\left\{\left(v_{i}, v_{j}\right) \mid\right.$ $\left.v_{i}, v_{j} \in V \wedge v_{i} \neq v_{j}\right\}$ be a finite set of links. Let $s, d \in V$ be two distinct nodes of the graph $G_{\mathrm{p}}$ and $|V|=N$, where $N$ is the number of nodes in the network. A path $P$ from $s$ to $d$ in the graph is a sequence of edges represented by $P=$ $\left\{\left(v_{1}, v_{2}\right), \ldots,\left(v_{k-1}, v_{k}\right)\right\}$, where $v_{1}=s, v_{k}=d$ and $\left(v_{i}, v_{i+1}\right) \in E$. Let $C_{l}$ be the cost function associated with the edges of the graph $G_{\mathrm{p}}$ defined as $C_{l}: V X V \rightarrow I$, where $I$ is the set of integers. 


\subsection{Auxiliary graph}

Let $G_{\mathrm{a}}=\left(V_{\mathrm{a}}, E_{\mathrm{a}}\right)$ represent the auxiliary graph of the given physical network topology graph $G_{\mathrm{p}}$, where $V_{\mathrm{a}}$ is the set of vertices and $E_{\mathrm{a}}$ is the set of edges. Let the wavelength channels available on a link be $\Lambda=\lambda_{1}, \lambda_{2}, \ldots, \lambda_{w}$, the total number of wavelengths being $w$. We construct $w$ copies of the physical network. Let the cost function associated with the edges of the auxiliary graph $G_{\mathrm{a}}$ be defined as $C_{\mathrm{a}}: V_{\mathrm{a}} X V_{\mathrm{a}} \rightarrow I$. The converter cost $C_{w}$ is greater than the longest wavelength continuous path in the network.

\section{Construct_auxiliary_graph() \\ begin \\ $V_{\text {temp }}=V, E_{\text {temp }}=E$ \\ Initialize $V_{\mathrm{a}}=\phi, E_{\mathrm{a}}=\phi$ \\ While $\left(V_{\text {temp }} \neq \phi\right)$ do \\ begin}

Choose the next element in $V_{\text {temp }}$; Call it $v_{e}$.

$V_{\mathrm{a}}=V_{\mathrm{a}} \cup\left\{v_{e}, v_{e}^{\prime}\right\}$

/* $v_{e}^{\prime}$ is a new supernode of $v_{e} * /$

$E_{\mathrm{a}}=E_{\mathrm{a}} \cup\left\{\left(v_{e}, v_{e}^{\prime}\right),\left(v_{e}^{\prime}, v_{e}\right)\right\}$

$C_{\mathrm{a}}\left(v_{e}, v_{e}^{\prime}\right)=0, C_{\mathrm{a}}\left(v_{e}^{\prime}, v_{e}\right)=0$

$V_{\text {temp }}=V_{\text {temp }}-\left\{v_{e}\right\}$

for $(p=1 ; p \leqslant w-1 ; p++)$

begin

I* $^{*}$ is the number of wavelengths $* /$

/* create a new vertex with node number $* 1$

$V_{\mathrm{a}}=V_{\mathrm{a}} \cup\left(v_{e+N * p}\right)$

$E_{\mathrm{a}}=E_{\mathrm{a}} \cup\left\{\left(v_{e}^{\prime}, v_{e+N * p}\right),\left(v_{e+N * p}, v_{e}^{\prime}\right)\right\}$

$C_{\mathrm{a}}\left(v_{e}^{\prime}, v_{e+N * p}\right)=0, C_{\mathrm{a}}\left(v_{e+N * p}, v_{e}^{\prime}\right)=0$ end

end

while $\left(E_{\text {temp }} \neq \phi\right)$ do

begin

choose the next edge in $E_{\text {temp }}$; call it $\left(v_{i}, v_{j}\right)$

$E_{\mathrm{a}}=E_{\mathrm{a}} \cup\left(v_{i}, v_{j}\right)$

$E_{\text {temp }}=E_{\text {temp }}-\left(v_{i}, v_{j}\right)$

$C_{\mathrm{a}}\left(v_{i}, v_{j}\right)=C_{l}\left(v_{i}, v_{j}\right)$

for $(p=1 ; p \leqslant w-1 ; p++)$

begin

$$
\begin{aligned}
& E_{\mathrm{a}}=E_{\mathrm{a}} \cup\left(v_{i+N * p}, v_{j+N * p}\right) \\
& C_{\mathrm{a}}\left(v_{i+N * p}, v_{j+N * p}\right)=C_{l}\left(v_{i}, v_{j}\right)
\end{aligned}
$$

end

end

$$
\begin{aligned}
& / * \text { add converter edges */ } \\
& V_{\text {temp }}=V \\
& \text { While }\left(V_{\text {temp }} \neq \phi\right) \text { do } \\
& \text { begin }
\end{aligned}
$$

Choose the next element in $V_{\text {temp }}$; Call it $v_{e}$. for $(p=0 ; p<w ; p++)$

for $(q=0 ; q<w ; q++)$

if $\left((p \neq q)\right.$ and $\left(v_{e}\right.$ has_a_converter $\left.)\right)$ then begin

$$
\begin{aligned}
& E_{\mathrm{a}}=E_{\mathrm{a}} \cup\left\{\left(v_{e+N * p}, v_{e+N * q}\right)\right\} \\
& C_{\mathrm{a}}\left(v_{e+N * p}, v_{e+N * q}\right)=C_{w}
\end{aligned}
$$$$
\text { end }
$$

end

end

\section{Problem}

We are given a physical network $G_{\mathrm{p}}(V, E, C, \Lambda)$ consisting of a finite set of nodes $V=\left(v_{1}\right.$, $\left.v_{2}, \ldots, v_{n}\right)$, a finite set of links $E=\left\{\left(v_{i}, v_{j}\right) \mid\right.$ $\left.v_{i}, v_{j} \in V \wedge v_{i} \neq v_{j}\right\}$, where each link is an ordered pair, and a set of wavelength channels $\lambda_{i} \in \Lambda$ where $i=1,2, \ldots, w$. The objectives are to,

- reduce the number of wavelength converters in the network;

- determine a near optimal placement of wavelength converters to reduce the overall blocking probability;

- reduce signal distortion by minimizing the number of cascaded conversions and limiting the range of wavelength conversion;

- to distribute the load uniformly by employing congestion control using dynamically varying state of the network.

\subsection{Assumptions}

- Each fiber link corresponds to a pair of unidirectional optical fibers.

- Each node can locally add or drop the optical signals. The required number of transmitters and receivers are available at each node.

- Each routing node is a reconfigurable switch. It may have full wavelength convertibility, limited wavelength convertibility or no wavelength convertibility. 
- Connection requests arrive at Poisson rate with exponential holding time. Connections requests can originate at any node and be wavelength routed to any other node in the network.

- The lightpath connection requests that are blocked do not return.

- The network topology is static and is not reconfigured during the computation.

- The first idle wavelength is assigned for a computed path (first-fit strategy).

\section{Analytical model}

An analytical model for the placement of wavelength converters in a ring network and an expression for the limited range wavelength conversion is derived in this section. Consider the example shown in Fig. 2, with the lightpaths established between nodes $1-2,3-4$, and $1-3$ on $\lambda_{1}$, $\lambda_{1}, \lambda_{2}$, respectively.

Each fiber supports two wavelengths, $\lambda_{1}$ and $\lambda_{2}$. If the next lightpath connection request is to be established between $2-4$, then this request can be set up only on a two hop path; $2-3$ on $\lambda_{1}$ and 3-4 on $\lambda_{2}$. This is possible only if the wavelength routing node has a wavelength converter at node 3 , otherwise, the call is blocked (Fig. 2). Thus, nodes with wavelength conversion capability reduce the call blocking probability and establish long lightpaths.

Consider a linear chain of $L$ hops as shown in Fig. 3. Let $w$ be the number of wavelengths available on a fiber link, $x$ be the probability that a wavelength is used on a link (wavelength occupancy), then $x w$ represents the expected number of busy wavelengths in a fiber link. It is assumed that a wavelength used on a link is statistically independent of other links and other wavelengths [29].

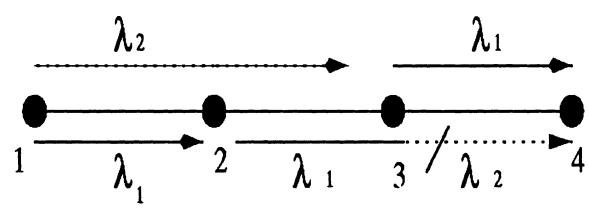

Fig. 2. Wavelength conversion at node 3 .

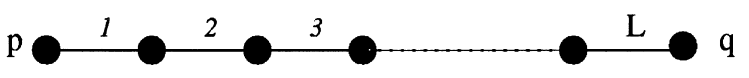

Fig. 3. A $L$ hop linear network.

In a network without wavelength converters, a lightpath connection request is blocked between $p$ and $q$ when every wavelength of the wavelength set $w$ is used on atleast one of the L links; the expression for blocking probability $P_{n w c}$ is obtained by

$P_{n w c}=\left[1-(1-x)^{L}\right]^{w}$.

In a network with wavelength converters at all the nodes, a lightpath connection request is blocked between $p$ and $q$ when every wavelength of the wavelength set $w$ is used on one of the L links; the expression for probability $P_{w c}$ is

$P_{w c}=1-\left(1-x^{w}\right)^{L}$.

Expressions (1) and (2) suggests that the blocking probability with and without wavelength converters increases with the increase in the number of hops. The blocking probability increases rapidly in the case of a network without wavelength converters and hence it is necessary to keep the path length small, i.e., small network diameter. On the other hand, blocking probability is much lower and fiber utilization is higher in the networks with wavelength converters.

Benefit of wavelength conversion increases with the length of a path and decreases with the increase in the number of wavelengths. It also depends on the number of wavelengths, network topology and the traffic matrix characteristics. In dense networks, the effectiveness of the wavelength converters is limited, whereas in a completely connected network, wavelength converters are not needed as all the lightpath request connections are single hops. On the other hand, the converters in a sparsely connected networks do not mix the connection requests well, causing load correlation in successive links.

Expressions (1) and (2) can be extended to multifibers where each link consists of $m$ fibers and the blocking probability without and with wavelength converters is given by expressions (3) and (4), respectively 
$P_{n w c}=\left[1-\left(1-x^{m}\right)^{L}\right]^{w}$,

$P_{w c}=1-\left(1-x^{m w}\right)^{L}$.

The multiplicity of links diminishes the gain obtained by the networks equipped with wavelength converters. By increasing the number of fibers, the network without wavelength converters are favored as the utilization of the network depends more on the multiplicity of links rather than the number of wavelengths.

\subsection{Placement of wavelength converters}

In [29], expressions for blocking probability are derived for a linear path with and without converters. This model along with Eqs. (1) and (2) can be used to determine the optimal placement of wavelength converters in a ring network for independent and uniform link loads. The objective is to position $C$ converters in a ring of $L$ nodes ( $L$ links) in order to minimize the blocking probability of an end-to-end call. Let the wavelength occupancy of all the links be equal, i.e., $x$. A segment is defined as a set of links between two successive wavelength converters. Therefore, $C$ converters in a ring will create $C$ segments and let the length of the segments be denoted by the segment length vector $S_{C}$ $=\left(s_{1}, s_{2}, s_{3}, \ldots, s_{C-1}, s_{C}\right)$, where $s_{i}$ is the link length of the $i$ th segment, $i=1,2, \ldots, C-1, C$ and $\sum_{i=1}^{C} s_{i}=L$. The probability of success $P\left(s_{i}\right)$ in each of the subsegment length $s_{i}$ can be determined from expression (5) and as follows:

$p\left(s_{i}\right)=1-\left(1-(1-x)^{s_{i}}\right)^{w}$.

Let the probability of success in a ring with segment length vector $S_{C}$ denoted by $P\left(S_{C}\right)$; then

$P\left(S_{C}\right)=\prod_{i=1}^{C} p\left(s_{i}\right)$.

Let the success probability for optimal placement of $C$ converters in a ring of $L$ hop path be denoted by

$P_{\text {opt }}(L, C)=P\left(S_{C}^{\mathrm{opt}}\right)=\max _{S_{C}} P\left(S_{C}\right)$,

where $S_{C}^{\text {opt }}$ is the optimum segment length vector. If $C$ divides $L$, then $s_{i}^{\text {opt }}=L / C$, where $i=$
$1,2, \ldots, C-1, C$ is the optimal segment length vector. In order to prove this, it is sufficient to show that

$$
\prod_{i=1}^{C} p\left(s_{i}^{\mathrm{opt}}\right)=\left[p\left(\frac{L}{C}\right)\right]^{C} \geqslant P\left(S_{C}\right)=\prod_{i=1}^{C} p\left(s_{i}\right)
$$

for any valid segment length vector. Taking logarithm of Eq. (7), the expression transforms to

$\ln p\left(\frac{L}{C}\right) \geqslant \frac{1}{C} \sum_{i=1}^{C} \ln p\left(s_{i}\right)$

where $s_{i}$ 's are the members of a valid segment length vector. The function $\ln p\left(s_{i}\right)$ is a concave function of the continuous variable $s_{i} \in(0, L)$. The concavity of the function is sufficient for the optimality of uniform placement of wavelength converters in a ring. The probability of success of a call in a ring with optimal placement of wavelength converters is given by

$P_{\mathrm{opt}}(L, C)=\left[p\left(\frac{L}{C}\right)\right]^{C}$.

This expression is exact only if $C$ divides $L$. If $C$ does not divide $L$, then the integral constraint on the segment lengths will make the above expression an upper bound on the probability of success. In such a case, the converters should be placed as uniformly as possible, that is, $z=L-y * C$ segments of length $y+1$ and $C-z$ segments of length $y$ where $y=\lfloor L / C\rfloor$. The blocking probability will be higher for random placement of wavelength converters [41]. This model can be extended to a path as proposed in [39].

Models provided by Barry and co-workers $[29,42]$ may be modified to derive expressions for the near optimal placement of wavelength converters in a large arbitrary mesh network which is a set of interconnected rings or conglomeration of a large number of paths. It is difficult to compute the optimal location of the wavelength converters in arbitrary mesh networks as the problem is NP complete [1]. The genetic algorithm can be applied for the near optimal placement of wavelength converters but it is computationally expensive [43]. In this paper, a heuristic is proposed for placement of wavelength converters in arbitrary mesh 
networks to achieve reduced call blocking probability of the network.

\subsection{Limited range wavelength conversion}

Wavelength conversion introduces distortion in the optical signal and the signal deteriorates rapidly in the process of cascading wavelength conversions. In optoelectronic wavelength converters, optical signal can be transformed from any wavelength to any other wavelength without degradation. All-optical wavelength converters are generally only capable of limited range wavelength conversion and the optical signal from input to output wavelength remains entirely in the optical domain. In FWM, wavelength conversion is a strong function of the input and output wavelengths and there is a significant degradation if the output signal is far away from the wavelength of the input signal. Hence, it is desirable to limit the range of wavelength conversion to reduce the optical signal distortion. The decrease in output power is nearly symmetrical about the input wavelength, i.e., a conversion from an input wavelength of $1520 \mathrm{~nm}$ to an output wavelength of $1530 \mathrm{~nm}$ produces nearly the same output power as a conversion from 1520 to $1510 \mathrm{~nm}$. The wavelengths used are within the $35 \mathrm{~nm}$ range $(1525-1560 \mathrm{~nm})$ of the low loss Erbium Doped Fiber Amplifier [44]. Limited wavelength conversion reduces the hardware costs of the optical switching nodes. Theoretical studies show that most of the reduction in the blocking probabilities is achieved using limited range wavelength conversion. Sparse or limited wavelength conversion can be of three types; (i) a limited number of nodes are provided with full range wavelength convertibility, (ii) a limited number of full range wavelength converters are placed at all nodes (share-per link or share-per-node), (iii) a limited range of wavelength conversion is only possible at the nodes. This paper explores the first and the third types of limited wavelength conversion.

\subsection{Adjacent wavelength conversion ( $A W C$ )}

The existing all-optical wavelength converters exhibit different levels of degradation of the signals for various combinations of input and output wavelengths. It is also a moot point whether to allow a larger number of lower loss wavelength conversions or accept a smaller number of highloss signal conversions so that the total signal degradation is below an acceptable level. A modified shortest path algorithm is used in an auxiliary graph to limit the number of cascaded wavelength conversions in [2].

The concept of adjacent wavelength conversion (AWC) model is introduced in [45]. Here, an incoming wavelength can be transformed to a small subset of nearby wavelengths at the output port i.e., an incoming wavelength can be converted only to a subset consisting of $d$ adjacent wavelengths on either side. This can be defined as $d$ adjacent wavelength conversion and the set of output wavelengths as convertible wavelength set. This model is different from the threshold model described in [38] where the boundary wavelengths are restricted from switching to adjacent wavelengths.

The AWC model for limited range wavelength conversion is depicted in Fig. 4. It consists of two links, each of which has $w$ wavelengths. The node 2 between the links 1 and 2 contains $w$ wavelength converters each of which can take a single input wavelength and convert to $d$ adjacent wavelengths on either side. A input signal entering on a wavelength $\lambda_{i}$ is converted into one of the wavelengths in the range of $\lambda_{(i-d)}$ to $\lambda_{(i+d)}$. For example, an input signal entering a node on the wavelength $\lambda_{2}$ can be transmitted by the wavelength converter on the second link on wavelengths $\lambda_{1}$ or $\lambda_{3}$ (the number of wavelengths per fiber link is four) apart from $\lambda_{2}$ for adjacent wavelength conversion degree $d=1$.

An expression for the blocking probability using limited range wavelength conversion can be derived from expressions (1) and (2). Let $\bar{\lambda}_{i, j}$ be the

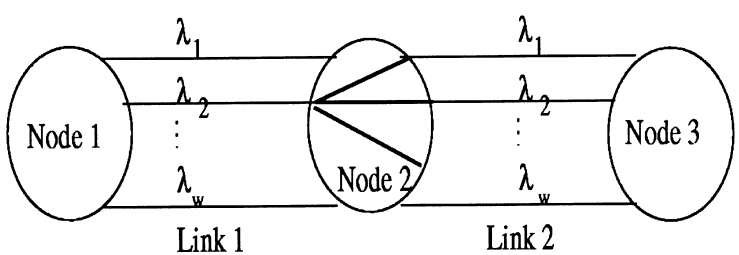

Fig. 4. Adjacent wavelength conversion at node 2. 
event that the path beginning with wavelength $\lambda_{i}$ on hop $j$ and terminating on the final hop $H$ is blocked. Then, the blocking probability between $j$ and $H$ is given by $p\left(\bar{\lambda}_{i, j}\right)$. Let $r(i)$ be the set of permissible output wavelengths for a limited range wavelength converter with the input wavelength $\lambda_{i}$; then define $\bar{\lambda}_{r(i), j+1}$ to be the event that all paths commencing on a wavelength within the set $r(i)$ are blocked from $j+1$ onwards. Hence, the probability of not establishing a lightpath from the hop $j+1$ to hop $H$ using any of the permissible wavelength conversions from wavelength $\lambda_{i}$ on the hop $j$ is given by $p\left(\bar{\lambda}_{r(i), j+1}\right)$. The blocking probability between the hop $j$ and hop $H$ from every wavelength on hop $j$ can be computed as follows:

$$
\begin{aligned}
& p \text { (path blocked from hop } j \text { to hop } H) \\
& \quad=p\left(\bigwedge_{i=1}^{w} \bar{\lambda}_{i, j}\right)
\end{aligned}
$$

The above equation can be computed by evaluating each of the $\bar{\lambda}_{i, j}$ elements as in [38]. A lightpath beginning on the wavelength $\lambda_{i}$ on hop $j$ is blocked either if all the permissible wavelength conversions from the wavelength $\lambda_{i}$ on hop $j$ lead to paths which are blocked or if the wavelength $\lambda_{i}$ is itself blocked on the hop $j$. The probability of the wavelength $\lambda_{i}$ being occupied on the hop $j$ is $x$ as defined earlier and it is independent of all other paths commencing from the hop $j$. On the other hand, the probability that all of the permissible conversions from wavelength $\lambda_{i}$ on hop $j$ leading to blocked paths are not in general independent of the other lightpaths beginning from the hop $j$, hence this probability cannot be evaluated directly. In fact, the blocking probability from hop $j$ is expressed in terms of the blocking probabilities from subsequent links within the path and the expression is recursively evaluated until the final hop is reached. It can be mathematically represented as follows:

$p$ (path blocked from hop $j$ to hop $H$ )

$$
\begin{aligned}
& =p\left(\bigwedge_{i=1}^{w} \bar{\lambda}_{i, j}\right) \\
& =p\left(\bar{\lambda}_{w, j} \wedge\left(\bigwedge_{i=1}^{w-1} \bar{\lambda}_{i, j}\right)\right)
\end{aligned}
$$

$$
\begin{aligned}
= & p\left(\left(\lambda_{w} \text { used on hop } j \vee \bar{\lambda}_{r(w), j+1}\right) \wedge\left(\bigwedge_{i=1}^{w-1} \bar{\lambda}_{i, j}\right)\right) \\
= & x \cdot p\left(\bigwedge_{i=1}^{w-1} \bar{\lambda}_{i, j}\right) \\
& +(1-x) \cdot p\left(\left(\bigwedge_{i=1}^{w-1} \bar{\lambda}_{i, j}\right) \wedge \bar{\lambda}_{r(w), j+1}\right) .
\end{aligned}
$$

The blocking probability $P_{\mathrm{B}}$ of the path is determined by computing the probability that no path exists from hop 1 to hop $H$. Thus,

$P_{\mathrm{B}}=p\left(\bigwedge_{i=1}^{w} \bar{\lambda}_{i, 1}\right)$

This is evaluated recursively from Eq. (10) in terms of $x$ and $H$. The probability of a path being blocked on a given wavelength on hop $H$ is given by $x$ with each path being independent. The above equation reduces to Eqs. (1) and (2), where $\bar{\lambda}_{r(i), j+1}=\bar{\lambda}_{i, j+1}$ (no wavelength conversion) and $\bar{\lambda}_{r(i), j+1}=\bigwedge_{i=1}^{w} \bar{\lambda}_{i, j+1} \quad$ (full range wavelength conversion)

Eq. (10) allows us to analyze the blocking probability for a path with limited range wavelength conversion. This can also be used to describe wavelength converters which use tunable transmitters with limited tuning ranges. If we allow one wavelength either side of the input wavelength, the allowed output wavelengths from the input wavelength $\lambda_{i}$ on link $j$ are $\lambda_{\max (i-1,1)}, \lambda_{i}$, and $\lambda_{\min (i+1, w)}$, for $1 \leqslant i \leqslant w$. In a general case, the conversions of $d$ wavelengths are permitted on either side of the input wavelength, then permitted output wavelengths on the succeeding hop will range from $\lambda_{\max (i-d, 1)}$, to $\lambda_{\min (i+d, w)}$. Thus

$\bar{\lambda}_{r(i), j+1}=\bigwedge_{l=\max (i-d, 1)}^{\min (i+d, w)} \bar{\lambda}_{l, j+1}$.

The degree of wavelength conversion, $D$, is defined as follows:

$D=\frac{100 d}{(w-1)} \%$.

If there are four channels per fiber link (i.e., $w=4$ ) and the wavelength conversion permissible is one wavelength on either side of the input wavelength 
$(d=1)$, the degree of wavelength conversion is $D= \pm 33.33 \%$. It indicates that the FWM limited range wavelength converter can convert either side from a given input wavelength by $D \%$ of the total range of wavelength conversion. The full range wavelength conversion is given by $D= \pm 100 \%$ and no wavelength conversion implies $D=0 \%$.

\subsection{Analytical results of blocking probabilities}

Eq. (10) can be used to compute the blocking probability for a particular path as a function of utilization $x$ and the degree of conversion $d$. The blocking probability versus the utilization for a two hop path for four wavelengths per fiber link is shown in Fig. 5. For example, when the utilization is $x=0.18$, the blocking probability is $12 \times 10^{-3}$ with no conversion and reduces almost by an order of magnitude to $2 \times 10^{-3}$ with full range wavelength conversion. Fig. 5 shows that there is a significant improvement in performance for all values of $x$ using limited range wavelength conversion. With $33 \%$ wavelength conversion range, the blocking probabilities close to that of full range wavelength conversion is obtained and if the degree of conversion is increased to $66 \%$, the difference in the performance of limited range wavelength conversion and full range wavelength conversion reduces further.
In multihop paths, there is a steep rise in the blocking probability without wavelength converters as it is difficult to find a single wavelength that can be allocated over all hops. However, the blocking probability is significantly reduced if full wavelength conversion is permitted. Networks with wavelength converters are more 'fair' to the paths of varying length as the blocking probability is no longer a significant function of the path length [1]. The analysis of limited range converters in two hop paths can be extended to multihops.

\section{Algorithms}

\subsection{Dynamic lightpath establishment}

This algorithm computes the shortest path using which a lightpath connection is setup dynamically between the source $s$ and destination $d$ in the auxiliary graph $G_{\mathrm{a}}$ (discussed in the previous chapter) by considering the network state. Appropriate weights are assigned to the link edges and converter edges based on the delay in fiber links and the cost of wavelength conversion respectively to ensure a minimum multihop path between an $s-d$ pair.

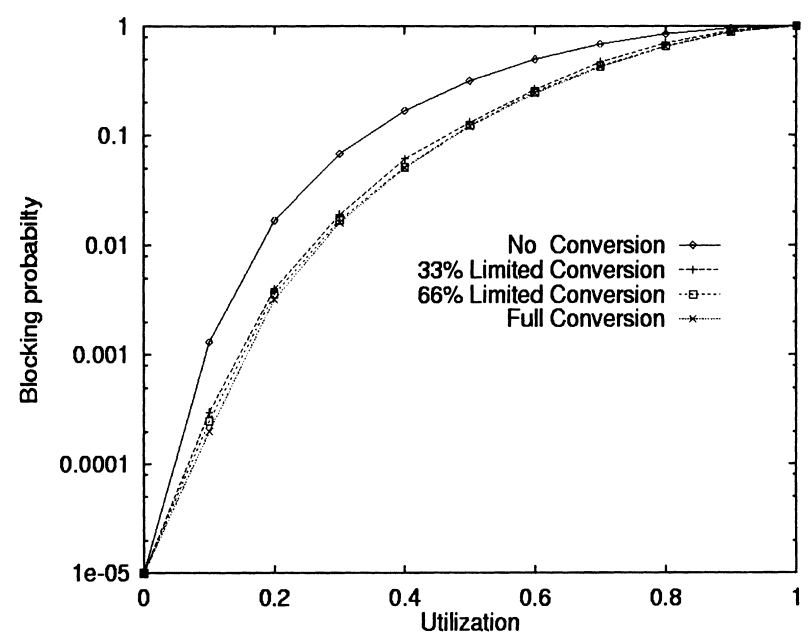

Fig. 5. Blocking probability for two hops with limited range wavelength conversion, four wavelengths. 


\subsection{Placement of limited range converters}

The placement of wavelength converters for SLE in a ring network has been explored in [22]. In the case of DLE, the limited range wavelength converters are placed uniformly as derived in the expression earlier.

\subsubsection{Ring network}

- Limited range wavelength converters are placed at a distance of $L / C$ in a ring network for uniform traffic with $L$ links and $C$ converters if $C$ divides $L$.

- Limited range wavelength converters are placed as uniformly as possible in a ring network, i.e., $z=L-y * C$ segments of length $y+1$ and $C-z$ segments of length $y$ where $y=\lfloor L / C\rfloor$, if $C$ does not divide $L$.

\subsubsection{Arbitrary mesh network}

The placement of wavelength converters in arbitrary mesh network is a NP complete problem [1]. A large number of lightpaths may transit through the nodes with high degree and hence wavelength conversions may be inevitable at these nodes [3]. In order to determine the placement of wavelength converters in arbitrary mesh networks, we first obtain certain traffic parameters by simulation assuming full convertibility at all the nodes. The parameters are, the number of lightpaths passing through a node, the number of wavelength conversions performed at a node and the average length of the lightpaths passing through the node. These traffic statistics for the 14-node NSFNET, 30-node INET, 28-node USA long haul network and 19-node EON are computed at 120 Erlangs load and this pattern is similar over a wide range of loads. Simulations are carried out at 120 Erlang loads with full range wavelength converters at all nodes with Poisson arrivals and exponential holding time. These statistics enable us to place the wavelength converters in the most appropriate positions in the network. There is a strong correlation between the degree of a node and its transit traffic. The following heuristic is used for placement of wavelength converters in arbitrary mesh networks [41]. (a) Nodes with high transit traffic.

(b) Nodes which lie on long lightpaths.

(c) Nodes which convert a large number of optical signals.

The number of wavelength converters is limited to less than one-third of the nodes of the network. The number of wavelength converters can be reduced further in the case of large networks. More weightage is given to nodes which transit large number of signals (around 60-70\%).

\subsection{Adjacent wavelength conversion}

In the adjacent wavelength conversion model, the wavelength is assigned from a set of available wavelengths at the instance of the establishment of the connection for a path. Initially, a connection request is attempted using a single wavelength. If a single wavelength is not available, then a minimum wavelength conversion path with $d=1$ (conversion range) is chosen. We move on to conversion degree $d=2$ in case of failure to establish a connection with wavelength conversion range $d=1$. It is achieved by choosing the weights of the wavelength converter arcs in the auxiliary graph appropriately. This algorithm effectively reduces the signal distortion in the network.

\subsection{Congestion control}

Congestion factor is defined as the number of lightpaths passing through the heaviest loaded link,

Congestion $=\max \left(l_{i}\right)$,

where $l_{i}$ represents the utilization of the $i$ th fiber link. Congestion is controlled by dynamically changing the weights of certain arcs in the auxiliary graph, thus balancing the load in the network. When the arc corresponding to the $\lambda_{k}$ channel of a link $\left(v_{i}, v_{j}\right)$ is used for setting up a lightpath, the weights of the arcs in the auxiliary graph $G_{\mathrm{a}}$ corresponding to all the other wavelengths of the link $\left(v_{i}, v_{j}\right)$ that are not assigned are incremented. These weights are decremented by the same value when the lightpath is taken down. The increment or decrement values are computed using a linear or non-linear function based on the current weights 
of the arcs and the number of available wavelength channels. The difference between the maximum and the minimum number of wavelength channels utilized in the links of the network converges and results in lower blocking probability.

The function Adjust_link_wtts() is used to dynamically adjust the weights of the edges in the auxiliary graph $G_{\mathrm{a}}$. When a path $P$ is established, the weights of the edges in the auxiliary graph that correspond to the edges in the path are increased by a factor of $w t t$ and they are reduced by the same weight when the lightpath is released. The pseudocode for the congestion control algorithm is given below.

\section{Congestion_Control}

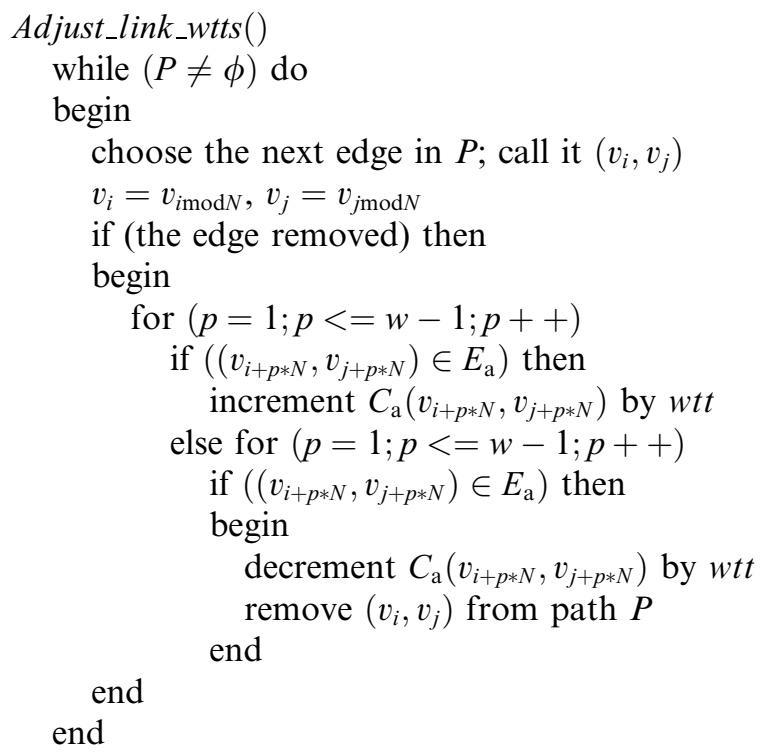

\section{Simulation}

\subsection{Performance evaluation}

This subsection describes the details of the simulation for determining the network performance by using the techniques proposed in the paper for dynamic lightpath establishment, placement of limited range wavelength converters and congestion control. The parameters of the physical network topology $\left(G_{\mathrm{p}}\right)$ such as the fiber link weights, and the wavelengths available per fiber link are taken as input and an auxiliary graph $G_{\mathrm{a}}$ is constructed. A request queue is necessary to hold the lightpath requests that are being serviced at any instant of time. It is a prioritized queue in which the lightpath requests are ordered according to their expirytime. The lightpath request source-destination pairs, arrival time and holding time according to a specified distribution are generated. The timer is incremented to the next lightpath request arrival time.

A path to route the lightpath request using ShortestPath algorithm is computed with the least number of wavelength conversions. If a path exists, enqueue the lightpath connection request, remove its path edges from the auxiliary graph and increment the weights of their corresponding edges as a congestion control measure. If a path does not exist, block the lightpath connection request. Lightpath connections (from request queue) which have been serviced are dequeued, their corresponding path edges in the auxiliary graph are replaced and decremented. The psuedocode of the simulation is given below.

\section{Simulation \\ begin}

Read_the_parameters_of_G $G_{\mathrm{p}}$

Initialize_req_q();

Construct_auxiliary_graph()

Initialize timer

while $($ timer $<$ max_time)

begin

$$
\begin{aligned}
& \text { timer }+=\text { Generate_arriv_time_- } \\
& \text { of_next_sd_pair }() \\
& \text { while }(\text { front_element_in_req_q } \rightarrow \\
& \text { expirytime }<\text { timer })
\end{aligned}
$$

begin $/ *$ element to be removed from the queue *1

Dequeue()

Restore_path_edges_in_auxiliary_graph() Adjust_link_wtts() end

Generate_next_sd_pair () request_hold_time $=$ Generate_hold_time () begin

I* path with minimum conversions*/

ShortestPath $(s, d)$

if $($ cost $\neq$ INFINITY $)$

$/ *$ INFINITY is a large constant */ 


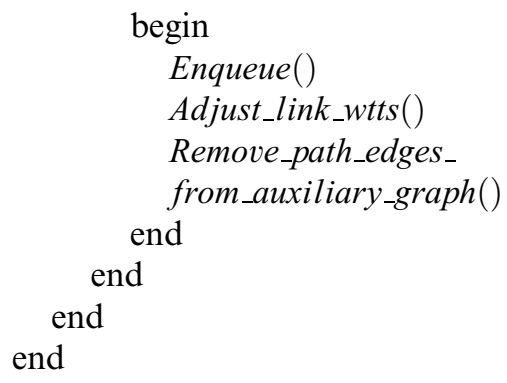

\subsection{Illustrative examples}

In a 12-node ring network, a single limited range wavelength converter is placed at node 7 for SLE. For DLE (Fig. 6), the converters are placed at 1,5 and 9 for uniform traffic if $C$ divides $L$; otherwise, the converters should be placed as uniformly as possible as derived in the expression earlier.

Fig. 7 shows the 14-node NSFNET and the placement of five wavelength converters. The reasons for their placement is given in the parenthesis. The wavelength converters are placed in the following order: 4(a,c), 9(a,c), 7(a,c), 6(a,c,b) and 11(a, c, b).

The 19-node European Optical Network (EON) is shown in Fig. 8. The wavelength converters are placed at nodes 1(a,c), 9(a,c), 17(b,c), 7(a,c), 4(a,c), 2(a, c, b) and 8(a).

The 28-node USA long haul network is shown in Fig. 9. The wavelength converters are placed at nodes 14(a,c), 12(a,c), 18(a,c), 17(a,b), 10(a,c), 5(a,b), 16(a,c), 26(a,b) and 9(a,b,c).

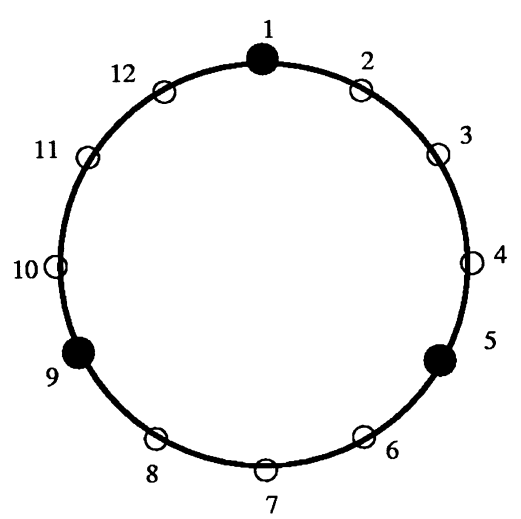

Fig. 6. 12-node ring network.

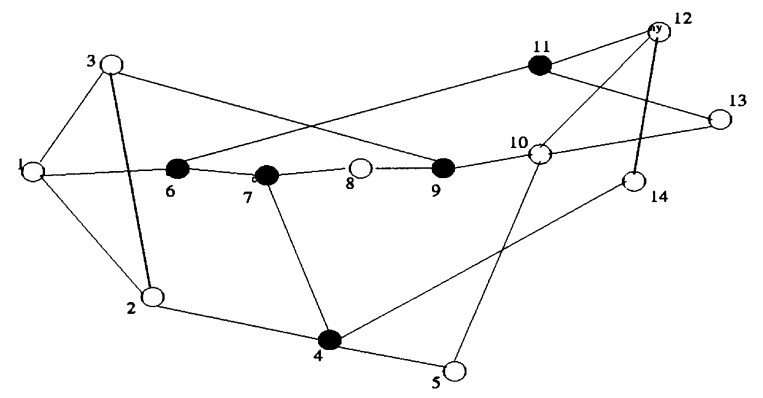

Fig. 7. 14-node NSFNET network with wavelength converters.

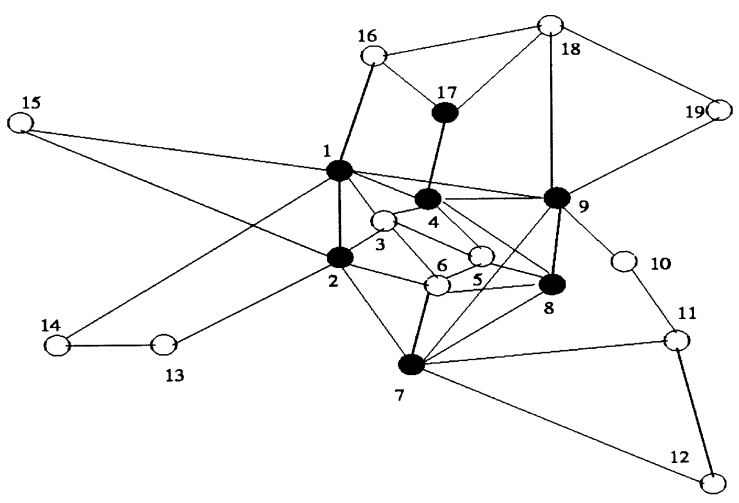

Fig. 8. 19-node European Optical Network (EON) with wavelength converters.

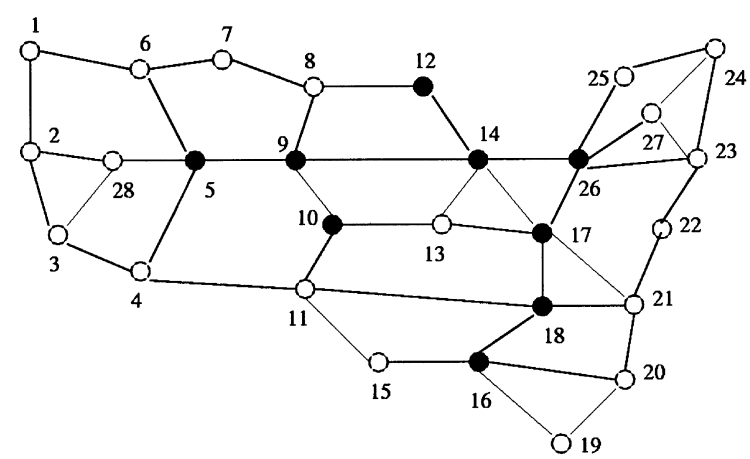

Fig. 9. 28-node USA long haul network with wavelength converters.

The placement of wavelength converters in the 30-node INET network is based on the heuristic described earlier in the algorithm. The wavelength converters are placed in the following order and 


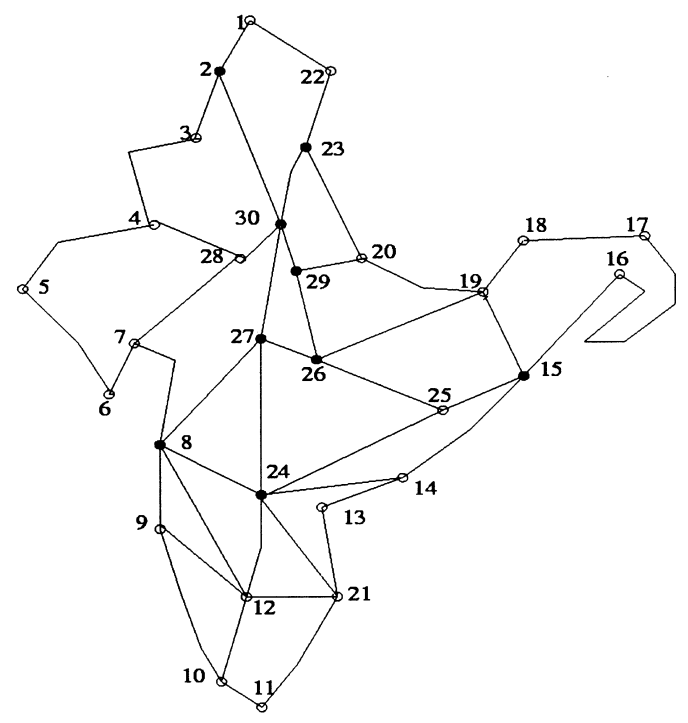

Fig. 10. 30-node INET network with wavelength converters.

reasons for placing nine wavelength converters are given in the parenthesis: 30(a,c), 2(b), 24(a,c), 23(b,c), 26(c), 27(a), 15(a,c), 29(b,c) and 8(a). Fig. 10 shows the placement of the converters for the INET.

\section{Numerical results}

Simulations have been used to examine the performance of placement of limited range wave- length converters in the 12-node ring, 14-node NSFNET, 28-node USA long haul network, 19node EON and 30-node INET mesh networks. The adjacency wavelength conversion model is used for limited range wavelength conversion. The placement of wavelength converters in a ring and mesh networks are given by the algorithm described earlier. The assumptions are the following: - An auxiliary graph is constructed for the given network as described earlier. See also [2,46].

- In the static routing, fixed routes have been used and in dynamic routing, connection is established using minimum number of hops, i.e., first, wavelength continuous path followed by minimum number of limited range wavelength conversion path.

- Uniform traffic distribution exists between all source-destination pairs.

In the 12-node ring network, the blocking probabilities for six and eight wavelengths have been plotted in Fig. 11 for SLE. It has been observed by us that the blocking probability decreases with the increase in the number of wavelengths per fiber links. A limited range wavelength converter placed in a ring gives the same performance as that of a network with full convertibility at all the nodes for the load $w$, where $w$ is the maximum number of channels utilzed in a link of the network. This is clearly evident in Fig. 11.

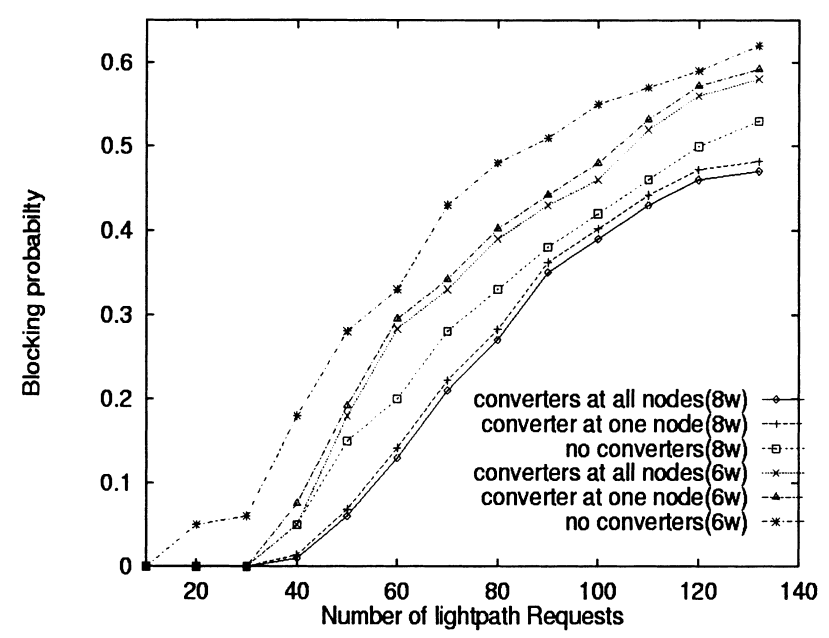

Fig. 11. Static lightpath establishment for 12-node ring with six and eight wavelengths. 
In the 12 node-ring network, the blocking probability performance is examined with respect to dynamic lightpath establishment. At lower loads, the blocking probability with wavelength converters is significantly lower and improves further at medium loads. At high loads, the networks without wavelength converters perform slightly better than the wavelength converter networks. This is due to certain long lightpaths that are established by the use of wavelength converters and the lightpaths with smaller path lengths which arrive later being blocked. Hence, though the fiber utilization is higher in wavelength convertible networks, the utilization of the fiber by long lightpaths leads to increase in the overall blocking probability of the wavelength convertible networks than the networks without wavelength converters. The blocking probability is higher when the nodes do not have wavelength converters; this is an account of lower utilization of the fiber links due to fragmentation problem, i.e., even-though different wavelength channels are available on the fiber links, the connection request cannot be established due to wavelength continuity constraint imposed by the non-availability of wavelength converters. Fig. 12 displays the above observations.

In the case of Dynamic lightpath establishment for ring networks, the increase in the number of wavelength converters beyond three improve the blocking probability performance only to a limited extent. This is evident in Fig. 13 which shows that there is only a marginal improvement after two or three converters are placed in the network. The blocking probabilities increase with increasing load and are examined for 20, 25 and 30 Erlang loads.

The 30-node INET network has been explored for the usage of limited range wavelength converters. It has been observed that the blocking probabilities are lower in the case of limited range wavelength converters at all the nodes. The blocking probabilities improve significantly with just $20 \%$ wavelength range conversion and the performance is very close to full range wavelength conversion with $40 \%$ wavelength range conversion. This is shown in Fig. 14. As the load increases, the overall blocking probability of the wavelength continuous network is slightly better for the reasons explained earlier. This phenomenon depends upon the network topology and the connectivity of the network.

In order to limit the use of expensive wavelength converters, they are placed only in a few nodes of the network, though the ideal choice is to have wavelength converters at all nodes. A trade-off between the number of wavelength

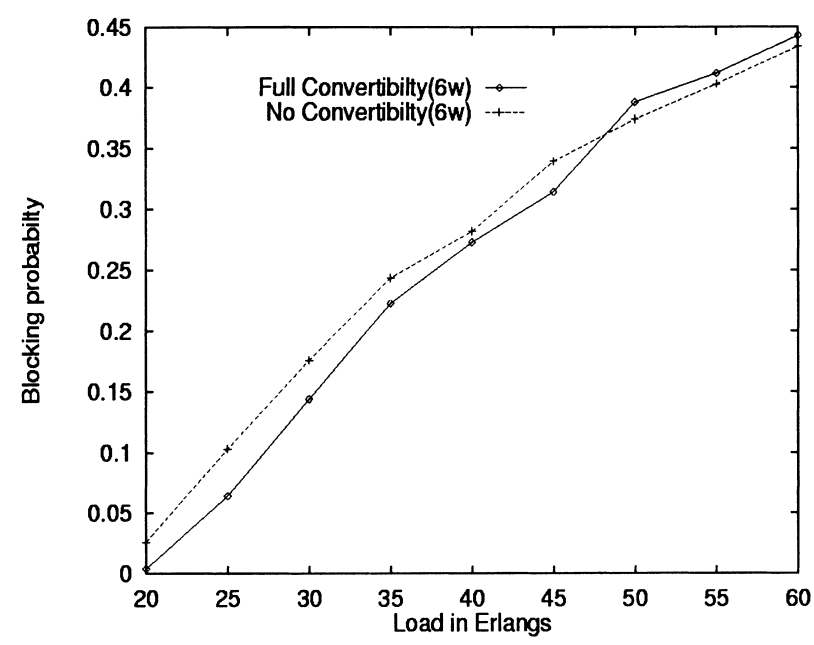

Fig. 12. Dynamic lightpath establishment for 12-node ring with and without conversion, six wavelengths. 


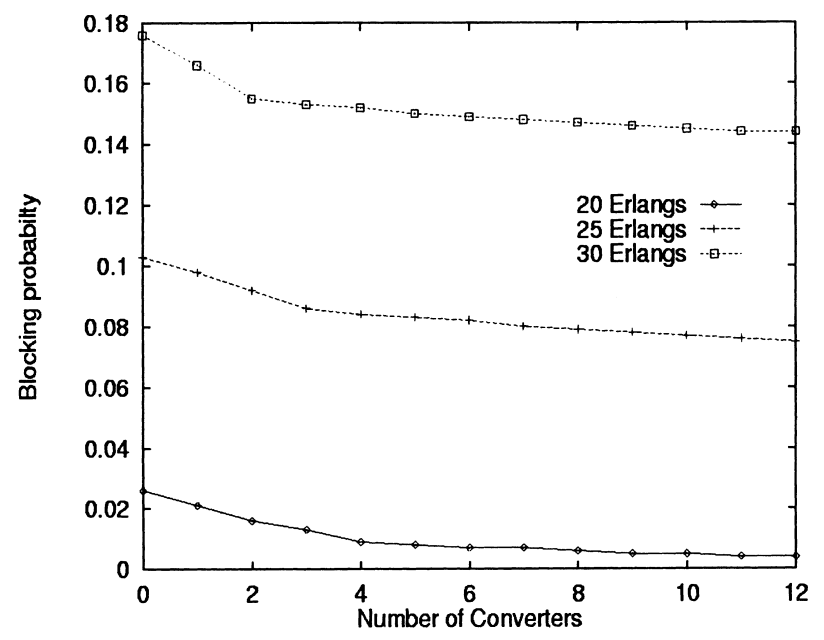

Fig. 13. 12-node ring with converters, six wavelengths.

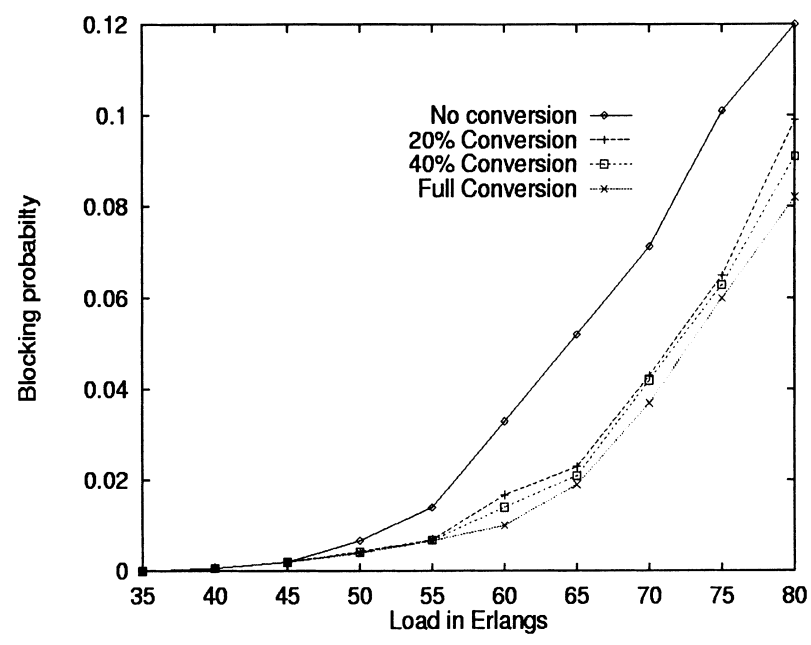

Fig. 14. Limited range converters at all nodes in 30-node INET.

converters and the blocking probabilities is explored here. The converters are placed according to the heuristics described earlier. The usage of only nine $40 \%$ limited range wavelength converters as shown in Fig. 10 gives the blocking probability performance very close to the full range wavelength converters placed at all the nodes of the network. In FWM wavelength conversion technique, the distortion is proportional to the distance of the conversion range. Hence, this technique of placing limited range wavelength converters reduces the optical signal distortion and also gives a good blocking probability performance. Thus, there are three important advantages of such a network model, i.e., blocking probability is reduced by using wavelength converters; the cost is reduced due to limited number of wavelength converters; signal distortion is reduced due to limited range conversion and minimum number of cascaded 
wavelength conversions. Fig. 15 shows that the blocking probabilities obtained with $20 \%$ limited range wavelength conversion, $40 \%$ limited range wavelength conversion, full range wavelength conversions at a few nodes and full range wavelength conversions at all the nodes are very close to each other.

In Fig. 16, the blocking probabilities for the 14-node NSFNET is carried out by using limited wavelength conversion only at five nodes of the network as shown in Fig. 7. It is interesting to note that the performance is similar to that of the 30 -node INET. The placement of the converters is based on the heuristic described in the algorithm. Another significant observation is that the network without wavelength converters has lower blocking probability at high loads (crossover effect). It is also observed that the crossover effect is a function of the number of wavelengths (at lower loads for smaller number of wavelengths),

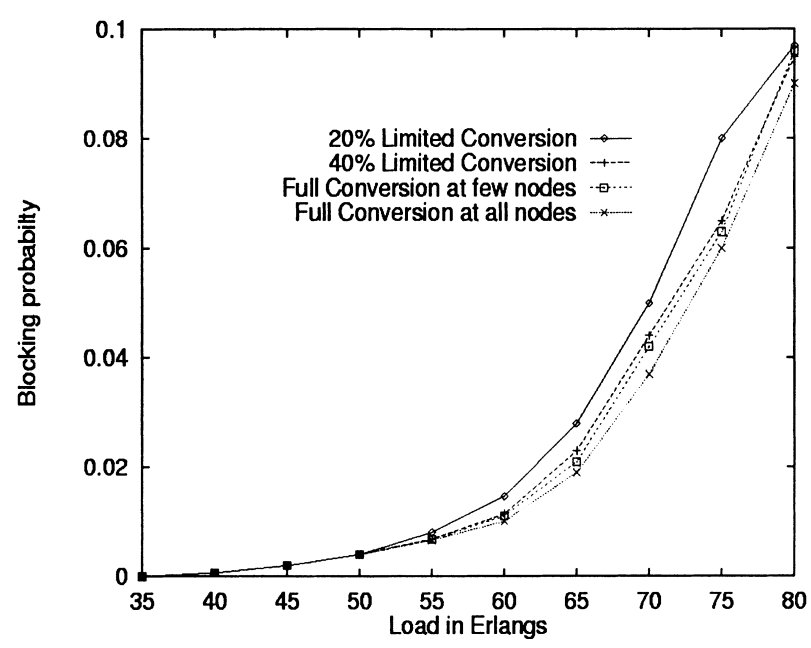

Fig. 15. Limited range converters at few nodes in 30-node INET.

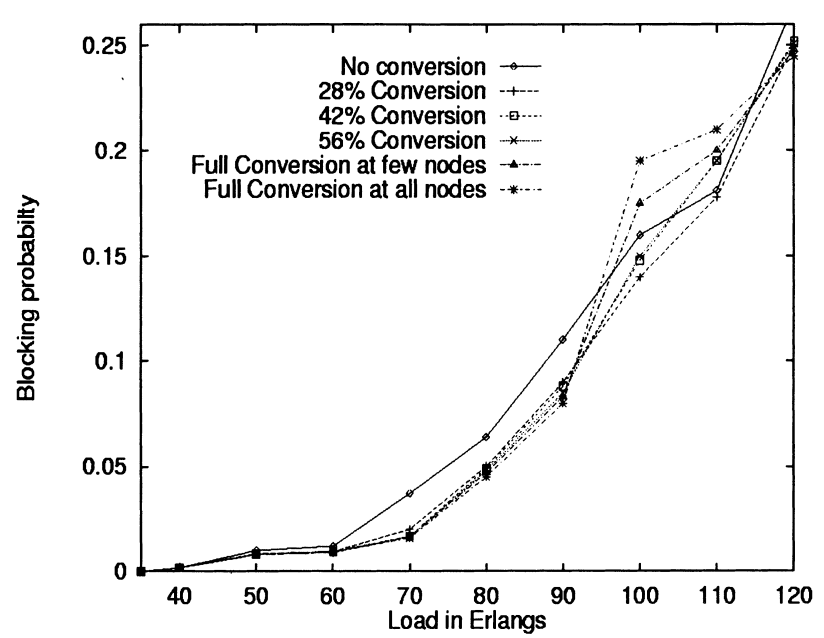

Fig. 16. Limited range converters in 14-node NSFNET network, eight wavelengths. 
size of the network and occurs only at higher blocking probabilities (greater than 15\%). The networks are normally designed for $1-2 \%$ blocking probability and hence this need not be considered [47]. This phenomenon occurs due to the sub-optimal algorithms used for routing and can be eliminated by using optimal routing algorithms. The crossover effect is observed at much lower loads in the 14-node NSFNET when compared to the 30-node INET. This is due to higher wavelength reuse in the 30-node INET. Finally, the number of limited range wavelength converters used can be varied depending on the required blocking probability performance of the network.

In Fig. 17, for 28-node USA long haul network, the blocking probability is plotted as a parameter with and without congestion control in the network. When a connection is established, the edges of the path are removed from the auxiliary graph and the weight of the corresponding edges are increased by a constant or a linear function or a non-linear function. The weights are decreased correspondingly when the edges are replaced after the connection is serviced. This algorithm ensures that the fiber links are utilized uniformly throughout the network contributing to lower blocking probability than when the congestion control is not used. Fig. 17 demonstrates the difference in the blocking probabilities with and without congestion control.

\section{Conclusions}

In summary, we have explored the impact of the limited range wavelength converters in WDM wavelength routed all-optical networks. At present, the optical converters are only capable of limited range wavelength conversion and the distortion of the optical signal is proportional to the distance of the wavelength conversion. An analytical model for the placement of wavelength converters in a ring network for uniform and independent load on links is derived. Wavelength converters in a ring network are placed as uniformly as possible. The placement of wavelength converters in arbitrary mesh networks is NP complete and hence a simple heuristic has been proposed. Limited range wavelength converters are placed at the nodes with high nodal degree which transit large amount of traffic and convert a large number of optical signals. In addition, nodes which lie on long lightpaths also need wavelength converters to give a performance reasonably close to that of a network with full range convertibility at all nodes. Hence, the limited range wavelength conversion at a limited number of nodes are viable

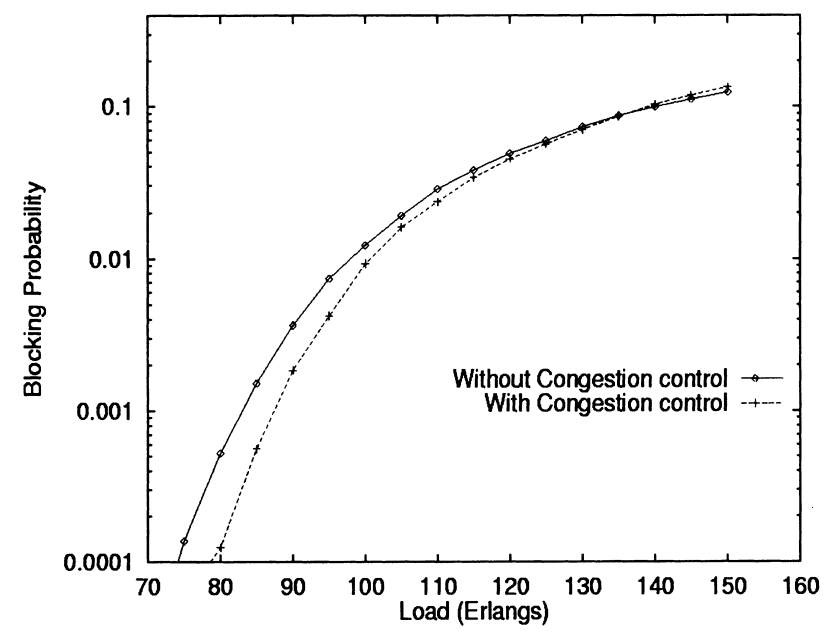

Fig. 17. Blocking probability with and without congestion control-adaptive approach. 
and cost effective. The placement of limited range wavelength converters in 14-node NSFNET, 19node EON, 28-node USA long haul network and 30-node INET are in agreement with our analysis. Share-per-link wavelength converters are used to increase the utility of the wavelength converters. This concept reduces the cost of the wavelength converters and the complexity of the switches. Blocking probability improves with the increase in the number of wavelengths. The dynamic lightpath establishment algorithm has been used to reduce the number of cascaded wavelength conversions and the wavelength assignment and conversion technique reduces the range of wavelength conversion resulting in lower signal distortion. The weights of the edges in the auxiliary graph are dynamically varied to control congestion in the network. Reduction in the number of wavelength conversions and controlling congestion reduces the overall delay in the network. To sum up, wavelength convertible networks are the state-of-the-art of the all-optical networks.

\section{References}

[1] K.C. Lee, V.O.K. Li, A wavelength-convertible optical network, IEEE Journal of Lightwave Technology 11 (1993) 962-970.

[2] K.R. Venugopal, A. Achutharam, P. Sreenivasa Kumar, An adaptive algorithm to reduce wavelength conversion and congestion in all-optical networks, in: Proceedings of the International Conference on Advanced Computing, Pune, India, December 1998, pp. 386-393.

[3] K.R. Venugopal, E. Ezhil Rajan, P. Sreenivasa Kumar, Performance analysis of wavelength converters in WDM wavelength routed optical networks, in: IEEE Proceedings of the International Conference on High Performance Computing, Chennai, India, December 1998, pp. 239-246.

[4] R. Ramaswami, Multiwavelength for computer communications, IEEE Communications Magazine 31 (1993) 78-88.

[5] R. Ramaswani, K. Sivarajan, Optical Networks: A Practical Perspective, Morgan Kaufmann, Los Altos, CA, 1998.

[6] B. Mukherjee, Optical Networks, McGraw-Hill, New York, 1997.

[7] K.R. Venugopal, P. Sreenivasa Kumar, Design issues in WDM wavelength routed optical networks, in: Proceedings of the International Conference on Advanced Computing, Chennai, India, December 1997, pp. 515-525.
[8] K.R. Venugopal, E. Ezhil Rajan, P. Sreenivasa Kumar, Impact of wavelength converters in wavelength routed alloptical networks, Computer Communications 22 (1999) 244-257.

[9] B. Mikkelsen et al., Interferometric wavelength converters for internal routing and wavelength slot interchange, in: Proceedings of the Photonics in Switching, Sendai, Japan, April 1996, pp. 166-167.

[10] M.J. O'Mahony, The potential of multivavelength transmission, in: Proceedings of the ECOC '94, Firenze, Italy, September 1994, pp. 907-913.

[11] S. Subramaniam, M. Azizoglu, A.K. Somani, Effect of wavelength converter density on the blocking performance of all-optical networks, in: Proceedings of the LEOS Annual Meeting '95, San Francisco, CA, October-November 1995, pp. 210-211.

[12] P. Cochrane, R. Heckingbottom, D. Heatley, The hidden benefits of optical transparency, IEEE Communications Magazine 32 (1994) 90-97.

[13] B. Mikkelsen et al., Wavelength conversion of high speed data signals, Electronic Letters 19 (1993) 1716-1718.

[14] K. Inoue et al., Influence of stimulated Brilliouin scattering on optimum length in fiber four wave mixing wavelength conversion, IEEE Photonic Technology Letters 7 (1995) 327-329.

[15] H.G. Weber et al., Four wave mixing for photonic switching, in: Proceedings of Photonics in Switching PMA5, Sendai, Japan, April 1996, pp. 160-161.

[16] T. Durhuus et al., High speed all-optical gating using twosection semiconductor optical amplifier structure, in: Proceedings of CLEO '92, Anaheim, CA, May 1992.

[17] R. Schnabel et al., Polarisation insensitive frequency conversion of a 10-channel OFDM signal using four wave mixing in a semiconductor laser amplifier, IEEE Photonic Technology Letters 6 (1994) 56-58.

[18] K. Stubkjaer et al., Optical wavelength converters, in: Proceedings of ECOC '94, Firenze, Italy, September 1994, pp. 635-642.

[19] R. Ramaswami, K.N. Sivarajan, Routing and wavelength assignment in all-optical networks, IEEE/ACM Transactions in Networking 3 (1995) 489-500.

[20] M. Kovacevic, A. Acampora, Benefits of wavelength translation in all-optical clear channel networks, IEEE Journal on Selected Areas in Communications 14 (1996) 868-880.

[21] O. Gerstel, P. Lin, G.H. Sasaki, A new angle on wavelength assignment in WDM rings: minimize system cost, not number of wavelengths, in: Proceedings of IEEE INFOCOM '98, San Francisco, CA, April 1998.

[22] O. Gerstel, R. Ramaswami, G.H. Sasaki, Benefits of limited wavelength conversion in WDM ring networks, in: Proceedings of the OFC '97, April 1997.

[23] O. Gerstel, R. Ramaswami, G.H. Sasaki, Fault tolerant multiwavelength optical rings with limited wavelength conversion, in: Proceedings of IEEE INFOCOM '97, Tokyo, Japan, April 1997. 
[24] O. Gerstel, R. Ramaswami, G.H. Sasaki, Cost effective traffic grooming in WDM rings, in: Proceedings of IEEE INFOCOM '98, San Francisco, CA, April 1998.

[25] R. Ramaswami, G.H. Sasaki, Multiwavelength optical networks with limited wavelength conversion, in: Proceedings of IEEE INFOCOM '97, Tokyo, Japan, April 1997, pp. 490-499.

[26] S. Banerjee, C. Chen, Design of wavelength-routed optical networks for circuit switched traffic, in: Proceedings of the GLOBECOM '96, London, 1996, pp. 306-310.

[27] A. Birman, Computing approximate blocking probabilities for a class of all-optical networks, IEEE Journal on Selected Areas in Communications 14 (1996) 852-857.

[28] I. Chlamtac, A. Farago, T. Zhang, Lightpath (wavelength) routing in large WDM networks, IEEE Journal on Selected Areas in Communications 14 (1996) 909-913.

[29] R.A. Barry, P.A. Humblet, Models of blocking probability in all-optical networks with and without wavelength changers, IEEE Journal on Selected Areas in Communications 14 (1996) 858-867.

[30] R.A. Barry, P.A. Humblet, On the number of wavelengths and switches in all-optical networks, IEEE Transactions on Communications 42 (1994) 868-880.

[31] K. Chan, T.P. Yum, Analysis of least congested path routing in WDM lightwave networks, in: Proceedings of the IEEE INFOCOM '94, October 1994, pp. 962-969.

[32] H. Harai, M. Murata, H. Miyahara, Performance of alternate routing methods in all-optical networks, in: Proceedings of the IEEE INFOCOM '97, Tokyo, Japan, 1997.

[33] S. Subramaniam, M. Azizoglu, A.K. Somani, All optical networks with sparse wavelength conversion, IEEE/ACM Transactions in Networking 4 (1996) 544-557.

[34] S. Subramaniam, A.K. Somani, M. Azizoglu, A performance model for wavelength conversion with non-Poisson traffic, in: Proceedings of the IEEE INFOCOM '97, Tokyo, Japan, 1997.

[35] O. Gerstel, S. Kutten, Dynamic wavelength allocation in WDM ring networks, in: Proceedings of IEEE INFOCOM '97, Tokyo, Japan, April 1997.

[36] G. Jeong, E. Ayanoglu, Comparison of wavelength interchanging and wavelength-selective cross-connects in multiwavelength all-optical networks, in: Proceedings of IEEE INFOCOM '96, San Francisco, CA, March 1996, pp. 156163.

[37] A. Mokhtar, M. Azizoglu, Adaptive wavelength routing in all-optical networks, IEEE/ACM Transactions on Networking 6 (1998) 197-206.

[38] J. Yates, J. Lacey, D. Everitt, M. Summerfield, Limited wavelength conversion in all-optical networks, in: Proceedings of IEEE INFOCOM '96, San Francisco, CA, April 1996, pp. 954-961.

[39] S. Subramaniam, M. Azizoglu, A.K. Somani, On the optimal placement of wavelength converters in wavelength routed networks, in: Proceedings of IEEE INFOCOM '98, San Francisco, CA, April 1998.
[40] V. Sharma, E.A. Varvarigos, Limited wavelength translation in all-optical WDM mesh networks, in: Proceedings of IEEE INFOCOM '98, San Francisco, CA, April 1998.

[41] K.R. Venugopal, M. ShivaKumar, P. Sreenivasa Kumar, A heuristic for placement of limited range wavelength converters in all-optical networks, in: Proceedings of the IEEE INFOCOM '99, New York, USA, March 1999, pp. 908-915.

[42] R. Barry, D. Marquis, Evaluation of a model of blocking probability in all-optical mesh networks without wavelength changers, in: Proceedings of SPIE East'95, Philadelphia, PA, October 1995, pp. 154-167.

[43] C. Vijayanand, M. ShivaKumar, K.R. Venugopal, P. Sreenivasa Kumar, Placement of wavelength converters in arbitrary mesh networks using genetic algorithm, Computer Communications 23 (2000).

[44] K.C. Lee, V.O.K. Li, Routing of all-optical networks using wavelengths outside Erbium-doped fiber amplifier bandwidth, in: Proceedings of IEEE INFOCOM '94, Los Angles, CA, April 1994.

[45] K.R. Venugopal, M. ShivaKumar, P. Sreenivasa Kumar, Placement of limited range wavelength converters in wavelength routed optical networks, in: Proceedings of the International Conference on Advanced Computing, Pune, India, December 1998, pp. 113-120.

[46] T.E. Stern, K. Bala, S. Jiang, J. Sharony, Linear lightwave networks performance issues, IEEE Journal of Lightwave Technology 11 (1993) 937-950.

[47] Tushart, K. Sivarajan, Limited range wavelength converters in wavelength routed optical networks, M.S. Thesis, Department of ECE, Indian Institute of Science, Bangalore, India, April 1998.

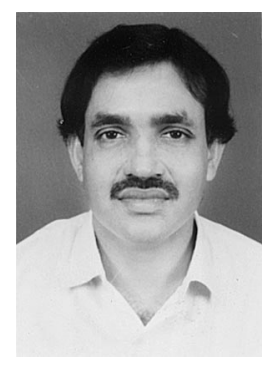

K.R. Venugopal obtained his Bachelor of Technology from University Visvesvaraya College of Engineering in 1979. He received his master's degree in Computer Science and Automation from Indian Institute of Science, Bangalore. He was awarded Ph.D. in economics from Bangalore University and Ph.D. in Computer Science from Indian Institute of Technology, Madras. He has a distinguished academic career and has degrees in Electronics, Economics, Law, Business Finance, Public Relations, Communications, Industrial Relations, Computer Science and Journalism. He has authored several books on Computer Science and Economics, which include Petrodollar and the World Economy, Programming with Pascal, Programming with Fortran, Programming with $\mathrm{C}$, Microprocessor Programming, Mastering $\mathrm{C}++$ etc. $\mathrm{He}$ has been serving as the faculty in the Department of Computer Science Engineering, University Visvesvaraya College of Engineering, Bangalore University, Bangalore for nearly two decades. His research interests include Computer Networks, Parallel and Distributed Systems, Object Oriented Languages and Database Systems.

M. ShivaKumar obtained his M.S. in Computer Science and Engineering from Indian Institute of Technology, Madras in 2000 . 


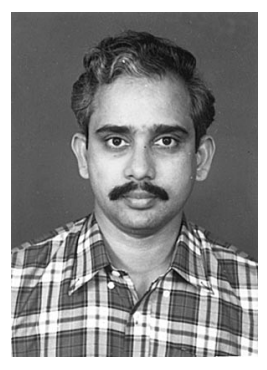

P. Sreenivasa Kumar obtained his Bachelor of Technology in Electronics and Communication Engineering from Sri Venkateswara University College of Engineering (Tirupati) in 1981. He earned Master of Engineering and $\mathrm{Ph} . \mathrm{D}$. both in Computer Science and Automation, Indian Institute of Science, Bangalore in 1984 and 1990, respectively. During 1984-85, he worked in the Indian Space Research Organization (ISRO) Satellite Center, Bangalore. He was also associated with the center for Development of Advanced Computing (C-DAC), Bangalore during 1990-93. Since June 1993, he has been working as a faculty member in the Department of Computer Science and Engineering, Indian Institute of Technology, Madras. He has research experience in the areas of algorithmic graph theory, parallel computing and computer networks. His current research activities are in the area of database system. 